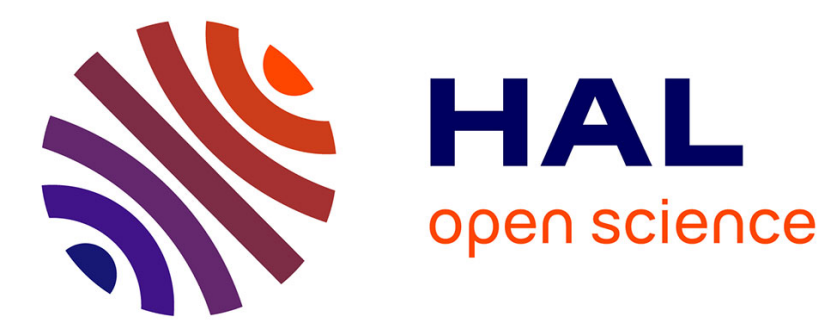

\title{
Thermocapillary manipulation of droplets using holographic beam shaping: Microfluidic pin ball
}

Maria Luisa Cordero, D.R. Burnham, Charles N. Baroud, D. Mcgloin

\section{To cite this version:}

Maria Luisa Cordero, D.R. Burnham, Charles N. Baroud, D. Mcgloin. Thermocapillary manipulation of droplets using holographic beam shaping: Microfluidic pin ball. Applied Physics Letters, 2008, 93 (3), pp.034107. 10.1063/1.2952374 . hal-01022812

\section{HAL Id: hal-01022812}

https://hal-polytechnique.archives-ouvertes.fr/hal-01022812

Submitted on $20 \mathrm{Jul} 2014$

HAL is a multi-disciplinary open access archive for the deposit and dissemination of scientific research documents, whether they are published or not. The documents may come from teaching and research institutions in France or abroad, or from public or private research centers.
L'archive ouverte pluridisciplinaire HAL, est destinée au dépôt et à la diffusion de documents scientifiques de niveau recherche, publiés ou non, émanant des établissements d'enseignement et de recherche français ou étrangers, des laboratoires publics ou privés. 


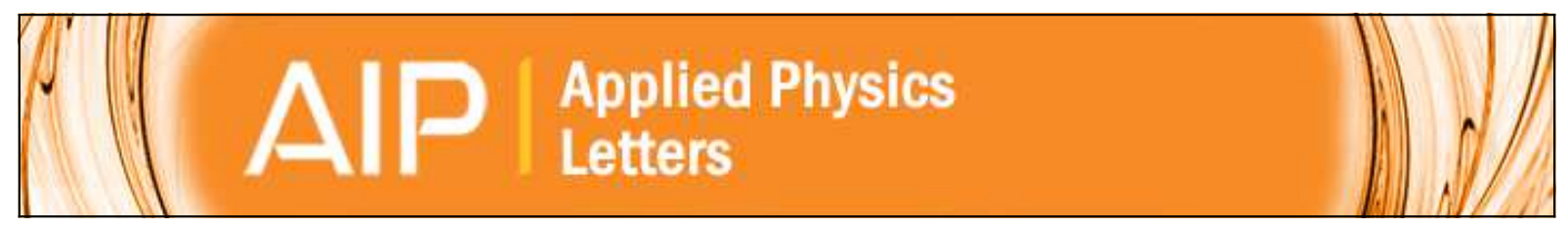

Thermocapillary manipulation of droplets using holographic beam shaping:

\section{Microfluidic pin ball}

Maria Luisa Cordero, Daniel R. Burnham, Charles N. Baroud, and David McGloin

Citation: Applied Physics Letters 93, 034107 (2008); doi: 10.1063/1.2952374

View online: http://dx.doi.org/10.1063/1.2952374

View Table of Contents: http://scitation.aip.org/content/aip/journal/apl/93/3?ver=pdfcov

Published by the AIP Publishing

\section{Articles you may be interested in}

Droplet deformation and manipulation in an electrified microfluidic channel

Appl. Phys. Lett. 103, 054101 (2013); 10.1063/1.4817008

Coplanar electrowetting-induced stirring as a tool to manipulate biological samples in lubricated digital microfluidics. Impact of ambient phase on drop internal flow patterna)

Biomicrofluidics 7, 044104 (2013); 10.1063/1.4817006

Micro-manipulation using rotational fluid flows induced by remote magnetic micro-manipulators J. Appl. Phys. 112, 064912 (2012); 10.1063/1.4754521

Novel method of generating water-in-oil(W/O) droplets in a microchannel with grooved walls

Biomicrofluidics 5, 014106 (2011); 10.1063/1.3567102

Dancing droplets onto liquid surfaces

Phys. Fluids 18, 091106 (2006); 10.1063/1.2335905

\section{A|P| Journal of}

Journal of Applied Physics is pleased to announce André Anders as its new Editor-in-Chief 


\title{
Thermocapillary manipulation of droplets using holographic beam shaping: Microfluidic pin ball
}

\author{
Maria Luisa Cordero, ${ }^{1}$ Daniel R. Burnham, ${ }^{2,3}$ Charles N. Baroud, ${ }^{1, a)}$ and David McGloin ${ }^{2, b)}$ \\ ${ }^{1}$ LadHyX and Department of Mechanics, Ecole Polytechnique, 91128 Palaiseau cedex, France \\ ${ }^{2}$ Electronic Engineering and Physics Division, University of Dundee, Nethergate, Dundee DD1 4HN, \\ United Kingdom \\ ${ }^{3}$ SUPA, School of Physics and Astronomy, University of St. Andrews, North Haugh, St. Andrews KY16 9SS, \\ United Kingdom
}

(Received 27 March 2008; accepted 17 May 2008; published online 24 July 2008)

\begin{abstract}
We demonstrate that holographically generated optical patterns offer greater flexibility for the thermocapillary control of water droplets than Gaussian spots; droplets can be stopped in faster flows while using less optical intensity when the surface tension variations are created by line patterns instead of single spots. Further, experiments are performed making use of variable light patterns to achieve controlled droplet routing in a four-way cross microfluidic channel. Finally, multiple droplet storage is demonstrated as well as changing drop order. (C) 2008 American Institute of Physics. [DOI: 10.1063/1.2952374]
\end{abstract}

Microfluidics and optical manipulation are two independently maturing technologies that enable advanced complementary studies of objects at the micron scale. In the case of optical manipulation, specifically optical tweezers, one of the major developments in recent years has been that of dynamic holographic optical trapping. ${ }^{1,2}$ These advances are currently providing increasingly complex possibilities in the control of droplets in the micrometer range. ${ }^{3,4}$ In parallel, microfluidics technology promises to provide automation through the miniaturization of biological and chemical systems. Many approaches are being explored toward this goal, of which the manipulation of droplets in microchannels is one of the most promising routes. ${ }^{5-7}$ In this "digital microfluidics," each droplet can be thought of as containing an independent reaction and one may want to perform either many copies of the same reaction, for example in the case of controlled chemical synthesis, ${ }^{8}$ or, instead, vary the parameters between droplets in order to explore a large number of combinations. ${ }^{9}$

The success of the latter case depends crucially on the ability to actively manipulate individual droplets as they flow through the microchannel network. Two main technologies have surfaced recently that allow the manipulation of these droplets. The first is based on application of an electric field to produce a force on the drop due to the dielectric contrast between the two liquids. This approach has been shown to work in sorting ${ }^{10}$ and merging droplets. ${ }^{11,12}$ The second technique varies the surface tension of the droplets locally, through the use of a focused laser beam. The flow that is generated by the thermocapillary effect was recently shown to produce a net force on the drop, experimentally and theoretically. ${ }^{13}$ This effect may be used to block the formation of drops, carry out simple routing, ${ }^{13}$ fuse them, synchronize them, or control their division. ${ }^{14}$

In this letter, we demonstrate how the combination of microfluidics with optical holographic techniques can be used to extend the possibilities of droplet manipulation. By taking advantage of the contactless nature of optical manipulation, we show how the use of different laser patterns allows

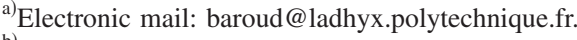

${ }^{b)}$ Electronic mail: d.mcgloin@dundee.ac.uk.
}

the implementation of complex operations, which are not possible using the current electrical forcing methods or single beam thermocapillary forces. Furthermore, the coupling between optical energy and thermocapillarity (optothermal forces) extends the possibilities of optical manipulation to drop sizes that are not accessible through optical forces alone. We begin by exploring the effect of the shape of the laser focus on the blockage of droplets. Indeed, the ability to vary the shape of the laser beyond a simple Gaussian beam provides an additional degree of freedom which can extend the limits of the technique.

Holographic beam shaping was employed to generate the desired patterns of light. ${ }^{15}$ The Gaussian beam from a 532 nm 4 W Laser Quantum Finesse continuous wave laser was expanded using a Keplerian telescope to entirely fill the short axis (768 pixels) of a Holoeye LCR-2500 spatial light modulator (SLM). Power was controlled with a polarizing beam cube and $\lambda / 2$ plate, while a second $\lambda / 2$ plate rotated the polarization to achieve optimal diffraction efficiency from the SLM. Two $4 f$ imaging systems demagnified the reflected beam such that the SLM was conjugate with the microscope objective's back aperture while slightly underfilling the pupil. Two objectives were used over the course of the experiments; the first was a Nikon $10 \times$ [numerical aperture $(\mathrm{NA})=0.25]$ and the second a Mitutoyo $10 \times(\mathrm{NA}$ $=0.26$ ), both of which focus the beam into the prefabricated microfluidic channels positioned on a three axis translation stage above the objective. The two objectives, in conjunction with custom built Köhler illumination and appropriate tube lenses, were also used to image the channels onto a Basler A602f firewire camera.

The phase of the beam incident upon the SLM was modified by displaying phase-only holograms whose optical Fourier transform gives the desired intensity pattern in the plane of the microfluidic channel. To produce the holograms, we implemented an adaptive-additive algorithm ${ }^{16}$ in custom written LABVIEW software, allowing the user to input an arbitrary 8 bit grayscale image of the desired intensity pattern. Aberration correction was combined with the hologram to remove the large amount of astigmatism inherent in the SLM. 

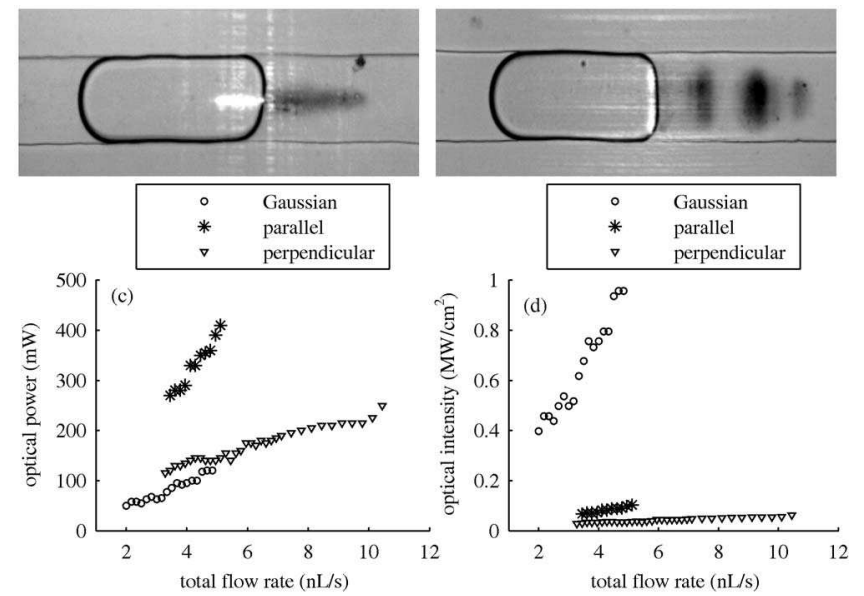

FIG. 1. Blocking a drop with different laser patterns: (a) a line parallel to the flow or (b) a line perpendicular to flow. (c) Minimum laser power and (d) minimum laser intensity required for blocking a drop.

The microfluidic chips were fabricated with molded PDMS (Sylgard 184, Dow Corning) using standard soft lithography techniques and sealed against a glass microscope slide. The dimensions of the channels ranged from 75 to $200 \mu \mathrm{m}$ in width and were $50 \mu \mathrm{m}$ in height. Oil (Hexadecane $+\operatorname{span} 80,2 \% \mathrm{w} / \mathrm{w})$ and an aqueous solution (water+ink 2\% v/v) are injected into the channel using syringe pumps. Dark blue Parker pen ink was added to the water in order to absorb the $532 \mathrm{~nm}$ light. Other absorbing dyes, or no dye, could be used depending on absorption requirements and the laser wavelength used. ${ }^{14}$

In order to investigate the effect of different light patterns on drops, we investigated the minimum optical power, $P_{\text {min }}$, required to block the advance of a drop, for three different shapes: a Gaussian spot with a $1 \mu \mathrm{m}$ waist, a straight line aligned with the flow direction, and a straight line perpendicular to the flow direction. Both lines were $2 \mu \mathrm{m}$ in width and $200 \mu \mathrm{m}$ in length. In these experiments, the chips had two oil inlets and one for the aqueous solution. Droplet size is determined by the ratio between the first oil flow rate $Q_{\text {oil }}^{(1)}$ and the water flow rate $Q_{\text {water, }}$, which were both kept constant. The second oil flow rate $Q_{\mathrm{oil}}^{(2)}$ was used to tune the total flow rate $Q_{\mathrm{tot}}=Q_{\mathrm{oil}}^{(1)}+Q_{\mathrm{oil}}^{(2)}+Q_{\text {water }}$, keeping the size of the droplets constant while their velocity varied with $Q_{\text {tot }}$.

The first observation, as the drops reach the laser beam, is that the water-oil interface adapts to the laser forcing, as seen in Figs. 1(a) and 1(b). When the line is parallel to the direction of flow, the front interface is flattened and the drop stops after advancing through a significant portion of the line. In the case of a line perpendicular to the flow direction, the surface of the drop is even flatter than in the previous case, taking on the shape of the line.

For each optical pattern used, the oil flow rate was varied from $Q_{\text {tot }} \sim 1$ to $11 \mathrm{~nL} / \mathrm{s}$ in increments of $0.17 \mathrm{~nL} / \mathrm{s}$. For each flow rate, we started from a high laser power and reduced it for successive drops, until the minimum power $P_{\text {min }}$ that still held the drops was reached. The maximum flow rate studied for each pattern was not limited by the laser no longer blocking the droplets, but rather by the water in the drops boiling.

The minimum laser power for each of the laser distributions is plotted as a function of the total flow rate in Fig. 1(c). It scaies approximately linearly with the total flow rate but

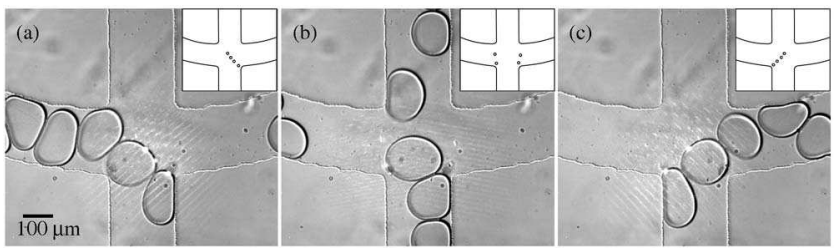

FIG. 2. Four light spots are aligned to sort droplets into either (a) the left-hand channel, (b) the center channel, or (c) the right channel. The insets show the positions of the holographically generated multiple Gaussian foci within the channel.

the slopes of the curves and the values of $P_{\min }$ differ for the three cases. The use of a line perpendicular to the flow allows the blocking of drops at higher flow rates, up to more than $10 \mathrm{~nL} / \mathrm{s}$. Conversely, even though a lower laser power is necessary to hold the droplets in the case of a Gaussian spot, it was not possible to hold droplets for flow rates higher than about $5 \mathrm{~nL} / \mathrm{s}$. This was also the case for the line parallel to the flow.

Moreover, if the pattern intensity is studied instead of the total laser power, the minimum intensity $I_{\min }$ necessary to block a drop is found to be several times higher for a Gaussian spot than for a line distribution [Fig. 1(d)]. The perpendicular line is found to block the drop for the lowest value of $I_{\min }$. Note that in the case of line patterns, the image is only fully formed in the focal plane of the microscope objective, i.e., measuring a few microns in depth. For the Gaussian spot, however, the pattern propagates through the whole sample.

We now consider how holographic beam shaping can extend single beam applications. ${ }^{13}$ The first application is droplet routing, actively sending droplets into different directions at a trifurcation. This is a precursor of sorting, one of the major applications in microfluidics. Making use of the ability to both dynamically switch the optical patterns projected into the microfluidic channel and the ability to create extended patterns (in this case four spots), we can deflect droplets through large angles and send them into preferred channels. This is shown using a four-way cross channel in Figs. 2(a)-2(c), ${ }^{17}$ which shows the droplets being moved to the left, straight, or to the right, respectively. The switching time of the droplets into a given channel is limited only by the update speed of the SLM, which ranges from 30 to $60 \mathrm{~Hz}$. It would be straightforward to extend this tech-

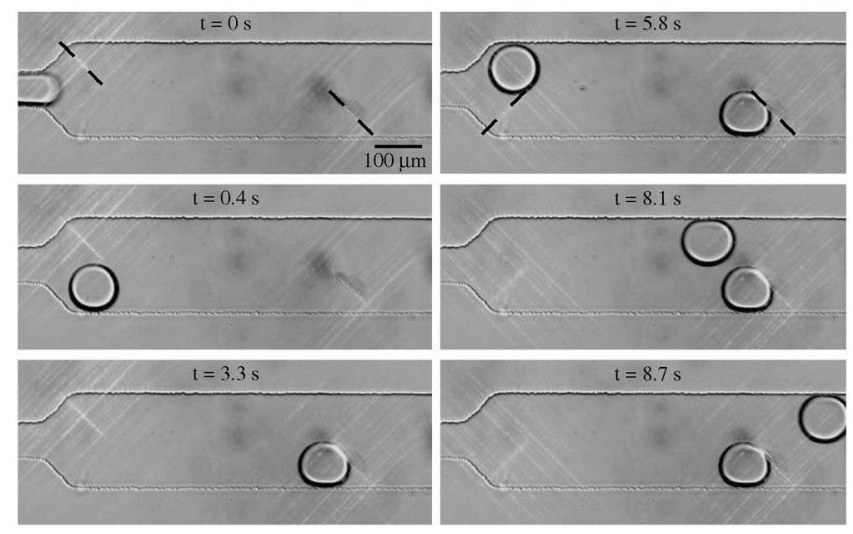

FIG. 3. Image sequence (left column followed by right column) showing how the drop order can be changed: The initial drop is sent down and held stationary then successive droplets are sent up. The dashed lines overlay the position of the laser patterns. 


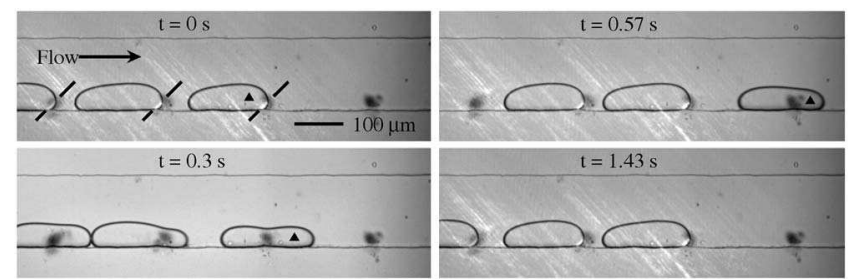

FIG. 4. A drop treadmill holds up to three droplets and can function as a first-in-first-out buffer memory. The triangle marks the same droplet in the different images (sequence is left column followed by right column), indicating its movement. The dashed lines overlay the position of the laser patterns. Total power in the sample plane is $\sim 475 \mathrm{~mW}$.

nique to active sorting, by including some video processing and combining it with the hologram switching (the holograms are precalculated and are merely changed based on which direction the droplets need to move in). One could imagine the sorting being based on droplet size, chemical composition, fluorescence measurements, or simply the contents of a drop.

The second example uses line patterns to store droplets at a given point in the channel while rerouting other droplets to move past the stored droplet. The first line upstream is set to move a droplet into one side of the larger channel. The droplet is then stored by the downstream line further along the channel. The first line is then changed so as to move subsequent droplets in the flow past the first droplet, as shown in Fig. $3 .^{17}$ Thus we can store and could interrogate the first droplet without the need to stop the flow, which is important in order to obtain longer interrogation times. Note that the ability to focus the laser to a small area on the drop allows real droplet-level manipulation, contrary to electrical fields which produce a uniform forcing on a region of the microchannel. ${ }^{11}$ This is what allows the drop order to be inverted in this case.

The third example, shown in Fig. $4,{ }^{17}$ is an extension of the second. Here we are able to trap several droplets at once, first one, then two, and finally three using three lines of light. Again this is in the presence of droplets flowing through the channel. We are then able to shuttle the droplets through the pattern, by turning the whole pattern on and off, so the first droplet is lost and the second droplet takes its place and so on. This allows large scale storage and controlled movement of many droplets simultaneously which may be useful for off-line analysis of many droplets, droplet reordering, or droplet "memory" applications.

In conclusion, we have shown that holographic beam shaping techniques have a number of advantages over conventional methods of inducing thermocapillary forces on droplets. In addition, extended patterns of light allow significant additional functionality over techniques using a single spot, which can lead to enhanced optical control in droplet microfluidics devices. Future extensions of our techniques include droplet sorting and droplet reordering, as well as more advanced studies into the role that extended optical patterns play in the forces induced.

The authors thank Emilie Verneuil for useful discussions. This work was partially funded by the British-Council PHC Alliance program. M.L.C. was funded by the EADS foundation and by MIDEPLAN. D.M. is a Royal Society Research Fellow.

${ }^{1}$ J. E. Curtis, B. A. Koss, and D. G. Grier, Opt. Commun. 207, 169 (2002).

${ }^{2}$ D. McGloin, Philos. Trans. R. Soc. London, Ser. A 364, 3521 (2006).

${ }^{3}$ D. R. Burnham and D. McGloin, Opt. Express 14, 4175 (2006).

${ }^{4}$ R. M. Lorenz, J. S. Edgar, G. D. M. Jeffries, Y. Q. Zhao, D. Mcgloin, and D. T. Chiu, Annu. Rev. Earth Planet Sci. 79, 224 (2007).

${ }^{5}$ H. Song, J. D. Tice, and R. F. Ismagilov, Angew. Chem., Int. Ed. 42, 768 (2003).

${ }^{6}$ H. Song, D. L. Chen, and R. F. Ismagilov, Angew. Chem., Int. Ed. 45, 7336 (2006).

${ }^{7}$ S.-Y. Teh, R. Lin, L.-H. Hung, and A. P. Lee, Lab Chip 8, 198 (2008).

${ }^{8}$ A. Günther and K. F. Jensen, Lab Chip 6, 1487 (2006).

${ }^{9}$ P. Laval, N. Lisai, J. B. Salmon, and M. Joanicot, Lab Chip 7, 829 (2007).

${ }^{10}$ K. Ahn, C. Kerbage, T. Hynt, R. M. Westervelt, D. R. Link, and D. A. Weitz, Appl. Phys. Lett. 88, 024104 (2006).

${ }^{11}$ K. Ahn, J. Agresti, H. Chong, M. Marquez, and D. A. Weitz, Appl. Phys. Lett. 88, 264105 (2006).

${ }^{12}$ C. Priest, S. Herminghaus, and R. Seemann, Appl. Phys. Lett. 89, 134101 (2006).

${ }^{13}$ C. N. Baroud, J. P. Delville, F. Gallaire, and R. Wunenburger, Phys. Rev. E 75, 046302 (2007).

${ }^{14}$ C. N. Baroud, M. R. de Saint Vincent, and J.-P. Delville, Lab Chip 7, 1029 (2007).

${ }^{15}$ D. R. Burnham, G. D. Wright, N. D. Read, and D. McGloin, J. Opt. A, Pure Appl. Opt. 9, S172 (2007).

${ }^{16}$ V. A. Soifer, V. Kotlyar, and L. Doskolovich, Iterative Methods for Diffractive Optical Elements Computation (Taylor \& Francis, London, 1997).

${ }^{17}$ See EPAPS Document No.E-APPLAB-92-041826 for movies of droplet manipulation. This document can be reached through a direct link in the online article's HTML reference section or via the EPAPS homepage (http://www.aip.org/pubservs/epaps.html). 